\title{
AS16-001. PREVALENCE AND PREDICTORS OF 3-MONTH FATIGUE IN PATIENTS WITH TIA AND MINOR STROKE: A CASE-CONTROL STUDY.
}

J.M. Ramirez-Moren ${ }^{01,2}$, P. Muñoz-Vega 3 , A.B. Constantino ${ }^{2}$, A. Roa ${ }^{2}$, J. Aguirre ${ }^{1}$, D. Peral ${ }^{4}$.

1. Extremadura University Medical School, Biomedical Sciences, Badajoz, Spain. 2. Hospital Universitario Infanta Cristina, Stroke Center. Neurology Department, Badajoz, Spain. 3. Hospital de Neurorehabilitación Casaverde, Neurology Area, Mérida, Spain. 4. Extremadura University Medical

School, Department of Medical-Surgery Therapeutics, Badajoz, Spain.

Background and Aims: The persistent and significant fatigue affects the daily life of major-stroke survivors, but the evidence suggests that transient ischemic attack (TIA) and minor stroke patients also experience significant fatigue. There are some population-based studies examining the prevalence of fatigue in patients with minor stroke and TIA very few studies of predictors of fatigue in these ones.

This study aimed to determine if fatigue prevalence in TIA patients is different to population controls, and to explore predictors of fatigue.

Results: The prevalence of substantial fatigue was $72.8 \%(23.5 \%$ in controls) and extreme fatigue was $20,7 \%(0.0 \%$ in controls $)$ in patients with minor stroke or TIA $(\mathrm{p}<0.0001)$. There are not was a clear association between fatigue and age (OR 1.00; IC95\%: 0.96-1.04). The only baseline variables independently associated with an increased risk of fatigue at 3 months after stroke were gender male, hypertension, dyslipidemia and physical inactivity.

Table. Clinical Characteristics Between Cases and Controls. Univariate Analysis.

\begin{tabular}{|c|c|c|c|}
\hline & $\begin{array}{l}\text { Controls } \\
(n=89)\end{array}$ & $\begin{array}{l}\text { Cases } \\
(n=92)\end{array}$ & $\mathbf{p}$ \\
\hline - Age, y, mean (SD) & $58.3(7,7)$ & $59.5(8.2)$ & 0.317 \\
\hline - Male sex, n(\%) & $37(43,0 \%)$ & $66(72.5 \%)$ & $<0.0001$ \\
\hline - Body Mass Index, mean (SD) & $27(4,1)$ & $28(3,9)$ & 0.166 \\
\hline - Rural residence, $\mathrm{n}(\%)$ & $55(61.8 \%)$ & $44(47.8 \%)$ & 0,073 \\
\hline \multicolumn{4}{|l|}{ Education } \\
\hline - Primary school and below (\%) & $20(22.5 \%)$ & $38(41.3 \%)$ & 0.005 \\
\hline - Middle and high school (\%) & $58(65.2 \%)$ & $51(55.4 \%)$ & \\
\hline - Bachelor and above (\%) & $11(12.4 \%)$ & $3(3.3 \%)$ & \\
\hline \multicolumn{4}{|l|}{ Physical activity previous stroke } \\
\hline - Sedentary (\%) & $14(15.7 \%)$ & $24(26.1 \%)$ & $<0.0001$ \\
\hline - Light intensity (\%) & $44(49.4 \%)$ & $62(67.4 \%)$ & \\
\hline - Moderate intensity (\%) & $31(34.8 \%)$ & $6(6.5 \%)$ & \\
\hline \multicolumn{4}{|l|}{ Medical history } \\
\hline - Hypertension (\%) & $30(33.7 \%)$ & $54(58.7 \%)$ & 0.001 \\
\hline - Hyperlipidaemia (\%) & $28(31.5 \%)$ & $40(43.5 \%)$ & 0.125 \\
\hline - Diabetes (\%) & $14(15.7 \%)$ & $28(30.4 \%)$ & 0.022 \\
\hline - Atrial fibrillation (\%) & $8(9.0 \%)$ & $11(12.0 \%)$ & 0.630 \\
\hline - Coronary heart disease (\%) & $1(1.1 \%)$ & $12(13.0 \%)$ & 0.002 \\
\hline - Smoking (\%) & $33(37.1 \%)$ & $59(64.8 \%)$ & $<0.0001$ \\
\hline - Depression (\%) & $11(12.4 \%)$ & $5(5.4 \%)$ & 0.121 \\
\hline - Family history of stroke (\%) & $27(30.3 \%)$ & $25(27.2 \%)$ & 0.743 \\
\hline - Family history of dementia (\%) & $24(27.0 \%)$ & $26(28.6)$ & 0.869 \\
\hline \multicolumn{4}{|l|}{ Fatigue } \\
\hline - FAS, mean (DS)* & $18,5(4.2)$ & $30.4(5.6)$ & $<0.0001$ \\
\hline - FAS physical, mean (SD) & $9,89(2.6)$ & $16.1(2.8)$ & $<0.0001$ \\
\hline - FAS mental, mean (SD) & $8.68(2.7)$ & $14.4(3.4)$ & $<0.0001$ \\
\hline - None (\%) & $65(76.5 \%)$ & $6(6.5 \%)$ & $<0.0001$ \\
\hline - Substantial fatigue (\%) & $20(23.5 \%)$ & $67(72.8 \%)$ & \\
\hline - Extreme fatigue (\%) & $0(0.0 \%)$ & 19 (20.7\%) & \\
\hline
\end{tabular}

Methods: Ninety-two consecutive patients with minor stroke or TIA were compared with 89 controls of the same age. The Fatigue Assessment Scale (FAS) was administered to both groups to compare prevalence.

The FAS is a 10-item general fatigue questionnaire to assess fatigue. Five questions reflect physical fatigue and 5 questions (questions 3 and 6-9) mental fatigue. FAS scores 10 - 21: no fatigue (normal); FAS scores 22 - 50: substantial fatigue. Two subgroups: (fatigue: scores 22-34 and extreme fatigue: scores $\geq 35$ )

The Minimal Important Difference (MCID) is at least 4 points or $10 \%$ change of the baseline value $(*)$.

Multivariate logistic regression analysis was used to determine predictors of fatigue development three months post-vascular event.

Table. Univariate logistic regression analysis was used to determine predictors of fatigue development

\begin{tabular}{lccc}
\hline Fatigue & OR & IC95\% & p \\
\hline Age & 1.002 & $0.965-1.040$ & 0.913 \\
Sex & 2.715 & $1.450-5.084$ & 0.002 \\
Physical inactivity & 2.151 & $1.307-3.541$ & 0.003 \\
Body Mass Index & 1.037 & $0.961-1.119$ & 0.345 \\
Hypertension & 2.140 & $1.153-3.974$ & 0.016 \\
Hyperlipidaemia & 2.731 & $1.404-5.312$ & 0.003 \\
Diabetes & 1.852 & $0.872-3.933$ & 0.109 \\
Atrial fibrillation & 0.912 & $0.347-2.393$ & 0.851 \\
Smoking & 2.544 & $1.370-4.725$ & 0.003
\end{tabular}

Table. Multivariate logistic regression analysis was used to determine predictors of fatigue development

\begin{tabular}{lcccc}
\hline Fatigue & OR & \multicolumn{2}{c}{ IC95\% } & p \\
\hline Age & 1.0 & 0.927 & 1.015 & 0.194 \\
Sex & 2.408 & 1.178 & 4.920 & $\mathbf{0 . 0 1 6}$ \\
Physical inactivity & 1.957 & 1.140 & 3.358 & $\mathbf{0 . 0 1 5}$ \\
Body Mass Index & 1.019 & 0.936 & 1.110 & 0.659 \\
Hypertension & 2.432 & 1.045 & 5.658 & $\mathbf{0 . 0 3 9}$ \\
Hyperlipidaemia & 2.224 & 1.021 & 4.842 & $\mathbf{0 . 0 4 4}$ \\
Diabetes & 0.601 & 0.221 & 1.634 & 0.318 \\
Atrial fibrillation & 0.934 & 0.306 & 2.854 & 0.905 \\
Smoking & 1.916 & 0.939 & 3.908 & 0.074
\end{tabular}

Figures. Relation between FAS score and Physical activityintensity, broken down by sex.
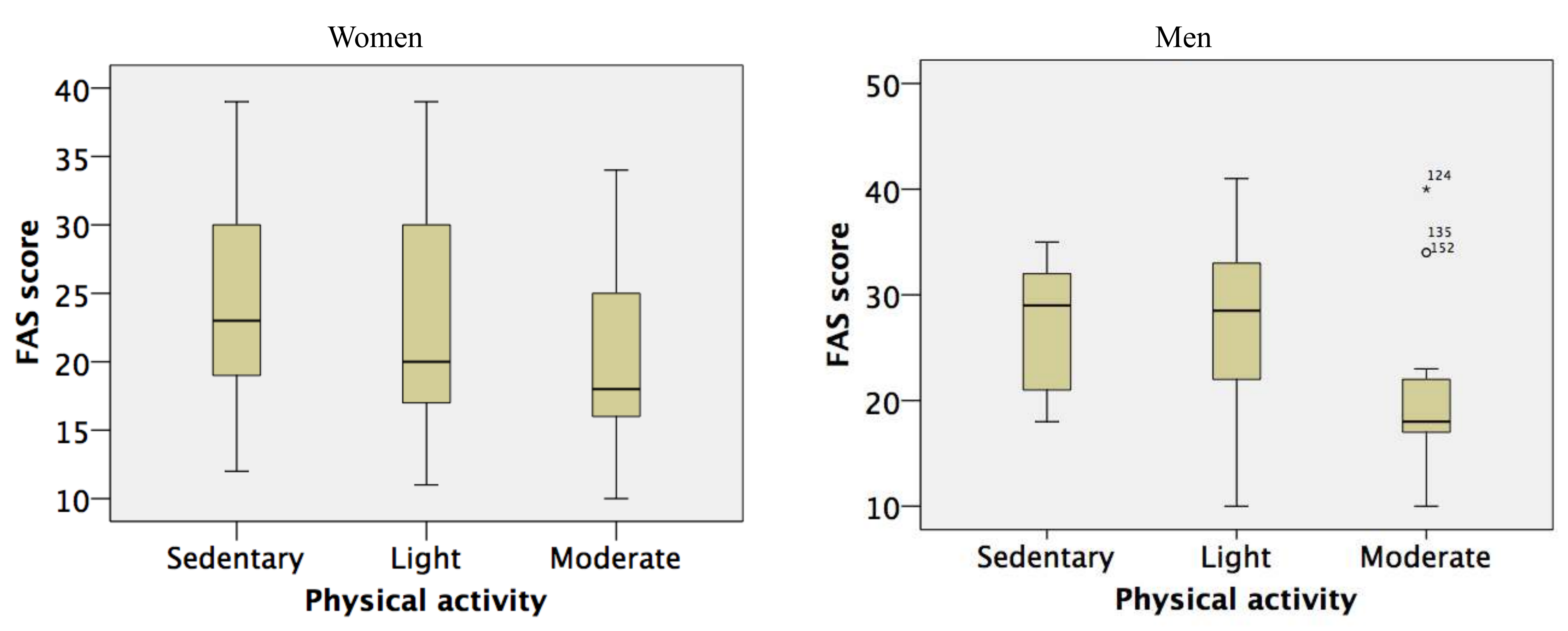

Conclusions: Minor stroke and TIA patients had higher prevalence of fatigue three months after stroke than the general population sample, which may be partly explained by the stroke population being in poorer health overall. Dyslipidemia, hypertension, physical inactivity, and gender male were independent clinical predictors of fatigue. 